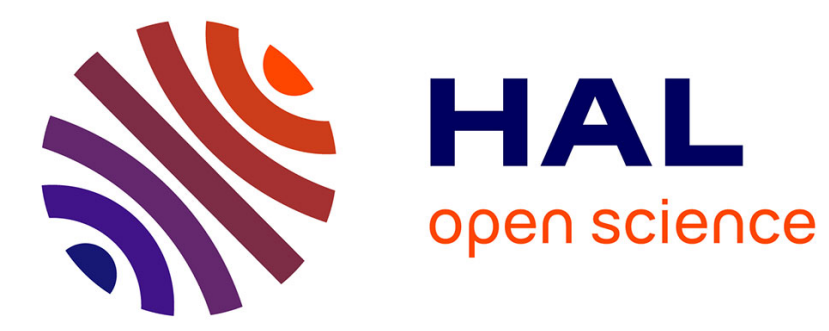

\title{
Quatrième partie. - Les propriétés magnétiques moyennes des métaux et alliages amorphes
}

\author{
J. Durand
}

\section{To cite this version:}

J. Durand. Quatrième partie. - Les propriétés magnétiques moyennes des métaux et alliages amorphes. Revue de Physique Appliquée, 1980, 15 (6), pp.1036-1042. 10.1051/rphysap:019800015060103600 . jpa-00244820

\section{HAL Id: jpa-00244820 https://hal.science/jpa-00244820}

Submitted on 1 Jan 1980

HAL is a multi-disciplinary open access archive for the deposit and dissemination of scientific research documents, whether they are published or not. The documents may come from teaching and research institutions in France or abroad, or from public or private research centers.
L'archive ouverte pluridisciplinaire HAL, est destinée au dépôt et à la diffusion de documents scientifiques de niveau recherche, publiés ou non, émanant des établissements d'enseignement et de recherche français ou étrangers, des laboratoires publics ou privés. 


\title{
Quatrième partie. - Les propriétés magnétiques moyennes des métaux et alliages amorphes
}

\author{
J. Durand \\ L.M.S.E.S. (L.A. 306), Institut Le Bel, 4, rue Blaise-Pascal, 67000 Strasbourg, France
}

1. Introduction. - L'état amorphe est un état métastable caractérisé à longue distance par le désordre tòpologique - et par là certaines de ses propriétés s'apparentent à celles de l'état liquide -, mais où un certain ordre à courte distance est préservé, - et par là certaines de ses propriétés reflètent celles de l'état cristallin correspondant. Aussi, l'état amorphe peut-il être défini selon deux différents modes d'approche : ou bien l'on part de l'état liquide en y surajoutant de l'ordre local (c'est la voie habituellement suivie dans l'étude des propriétés de structure ou de transport); ou bien l'amorphe est compris comme un cristal que l'on désordonne à l'extrême (ce point de vue est plus commode pour l'analyse des propriétés mécaniques ou magnétiques). Nous résumons les différents résultats obtenus sur les propriétés magnétiques moyennes des alliages métalliques amorphes à base de métaux de transition ou de terres rares, en nous attachant plus particulièrement à ceux qui permettent une comparaison avec des systèmes cristallins de composition identique ou voisine. Le cas des métaux de transition et du gadolinium est discuté en premier lieu. Les éléments de terres rares présentant des états ioniques non-S seront analysés de façon séparée, en raison des problèmes particuliers posés par les effets de gradient de champ électrique, l'anisotropie locale, et les valences anormales.

2. Le magnétisme des métaux de transition et du gadolinium en milieú amorphe. - 2.1 DE L'IMPURETÉ DILUÉE A L'ORDRE MAGNÉTIQUE A LONGUE DISTANCE. Les propriétés magnétiques d'alliages dilués de $\mathrm{Co}$, $\mathrm{Fe}, \mathrm{Mn}, \mathrm{Cr}$ dans des matrices paramagnétiques amorphes comme PdSi [1], PdP [2], PdB [3], NiPB [4], $\mathrm{Cu}-\mathrm{Zr}$ [5] ont été étudiées de façon relativement détaillée. Il ne semble pas que dans la plupart de ces études l'on ait pu atteindre des concentrations suffisamment faibles pour que l'on ait affaire à des régimes Kondo dilués en ce qui concerne le fer; il s'agirait plutôt de régimes de verres de spins. Les alliages de $\mathrm{La}-\mathrm{Au}$ avec le $\mathrm{Gd}$ se prêtent particulièrement bien à une analyse en termes de verres de spin jusqu'à des concentrations élevées (30\% de Gd) [6]. Contrairement à ce que l'on pourrait penser de prime abord, le très court libre parcours moyen électronique dans une matrice amorphe ne semble pas avoir pour effet d'élever la concentration critique entre le régime d'im- pureté diluée et le régime de verres de spin. Comme dans le cas cristallin, des concentrations de l'ordre de $1 \%$ de Fer [7] ou de Gd [6] correspondent déjà à des verres de spin concentrés où les spins interagissent entre eux sous forme d'amas. Pour des concentrations de $\mathrm{Gd}$ inférieures à $1 \%$ dans le cas de $\mathrm{La}-\mathrm{Au}$, on observe toutes les caractéristiques des verres de spin canoniques, et les paramètres que l'on peut déduire des propriétés supraconductrices [8] et magnétiques [9] ne mettent pas en évidence l'atténuation notable de l'interaction de Ruderman-Kittel que l'on attendrait du fait du libre parcours moyen très court [10]. La définition du libre parcours moyen pour les interactions magnétiques dans un amorphe mérite d'être reconsidérée [11].

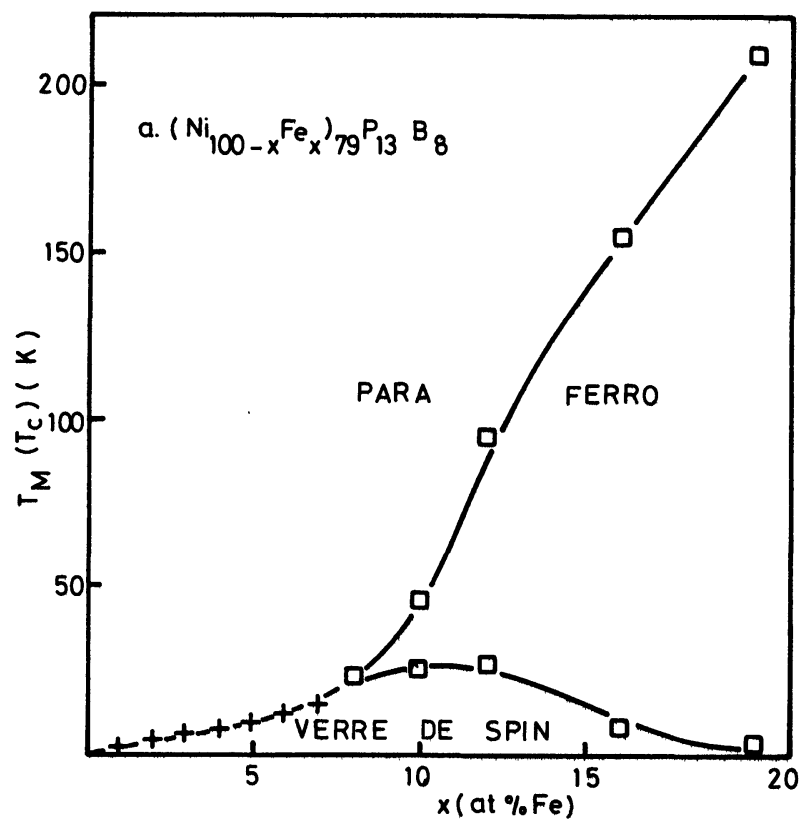

Fig. 1. - Variation des températures des maxima de susceptibilité $\left(T_{\mathrm{M}}\right)$ et des températures de Curie $\left(T_{\mathrm{c}}\right)$ en fonction de la concentration en Fer dans le système amorphe $\left(\mathrm{Ni}_{100-x} \mathrm{Fe}_{x}\right)_{79} \mathrm{P}_{13} \mathrm{~B}_{8}$. Pour $10 \leqslant x \leqslant 20$, les alliages, ferromagnétiques à haute température, connaissent une transition ferro-verre de spin à basse température (référence [17]).

[Susceptibility maximum temperatures $\left(T_{\mathrm{M}}\right)$ and Curie temperatures $\left(T_{\mathrm{c}}\right)$ as a function of $\mathrm{Fe}$ concentration in amorphous

$$
\left(\mathrm{Ni}_{100-x} \mathrm{Fe}_{x}\right)_{79} \mathrm{P}_{13} \mathrm{~B}_{8}
$$

alloys. For $10 \leqslant x \leqslant 20$, the alloys, which are ferromagnetic at high temperature, undergo a ferromagnetic-to-spin glass transition at low temperature (reference [17]).] 


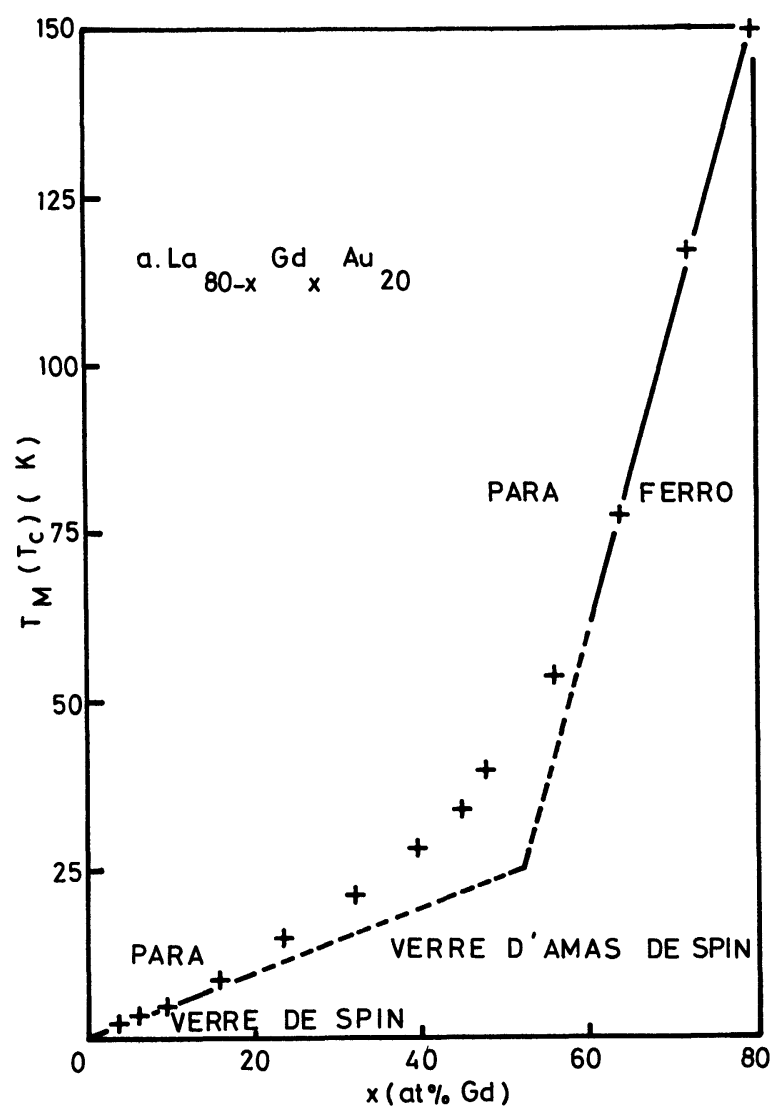

Fig. 2. - Températures des maxima de susceptibilité $\left(T_{\mathrm{M}}\right)$ et températures de Curie $\left(T_{\mathrm{c}}\right.$, pour $\left.x \geqslant 60\right)$ en fonction de la teneur en $\mathrm{Gd}$ dans le système amorphe $\mathrm{La}_{80-x} \mathrm{Gd}_{x} \mathrm{Au}_{20}$ (référence [6]).

[Susceptibility maximum temperatures $\left(T_{\mathrm{M}}\right)$ and Curie temperatures $\left(T_{\mathrm{c}}\right.$, for $\left.x \geqslant 60\right)$ as a function of $\mathrm{Gd}$ content in amorphous $\mathrm{La}_{80-x} \mathrm{Gd}_{x} \mathrm{Au}_{20}$ alloys (reference [6]).]

Pour des concentrations suffisamment importantes en impuretés magnétiques, on observe l'apparition d'un ordre ferromagnétique à longue distance. Cette approche du ferromagnétisme pour des concentrations croissantes d'impuretés a été étudiée soit dans des systèmes où l'impureté porte un moment au sens de Hartree-Fock (Fe dans PdP [2] ou dans NiPB [4], Gd dans LaAu [6]), soit dans le cas où l'impureté diluée est non magnétique, mais où le moment apparaît pour un environnement donné autour de l'impureté (Co dans NiPB [4], ou les systèmes binaires NiP [12], YNi [13], YFe [14], FeSi, FeGe, FeSn [15] ou ternaires, comme NiPB [16]). Dans tous ces systèmes, le ferromagnétisme apparaît de façon inhomogène, comme dans les systèmes cristallins voisins. Pour des concentrations d'impuretés inférieures à la concentration critique pour l'apparition d'un ordre magnétique à longue distance, ces alliages présentent les caractéristiques des verres de spin concentrés (ou mictomagnétiques) : les spins sont ordonnés en amas dont la taille moyenne croît rapidement quand on s'approche de la concentration critique. Comme dans le système canonique $\mathrm{Au}-\mathrm{Fe}$, ces amas peuvent persister à l'intérieur d'un système où la percolation est réalisée pour un certain nombre de spins et donner lieu à une anomalie de susceptibilité de type verres de spin pour des températures inférieures à la température de Curie correspondant à la percolation (voir Fig. 1). Ces inhomogénéités magnétiques ne sont pas propres à l'état amorphe, mais il semble évident que le désordre topologique tend à en élargir la distribution et à accroître le domaine de concentration où elles se manifestent.

2.2 LES AMORPHES FERROMAGNÉTIQUES A BASE DE MÉTAUX DE TRANSITION ET DE GADOLINIUM. - L'effet du désordre structural semble $a$ priori facile à circonscrire dans ces alliages. On suppose de façon habituelle que les interactions magnétiques sont à courte distance. La diversité des environnements possibles pour un atome de métal de transition (ou de Gd) ainsi que les fluctuations dans les distances interatomiques ont pour conséquence de créer une distribution des paramètres d'échange. La valeur moyenne de l'échange n'est que peu affectée par le désordre. Par ailleurs, l'effet de la distribution des gradients de champ électrique est supposé négligeable comparé à la distribution sur l'échange. Le caractère quelque peu arbitraire de ces hypothèses sera discuté plus tard. Nous analysons l'effet du désordre sur le moment à saturation à $0 \mathrm{~K}$, sur les transferts de charge, sur la dépendance en température de l'aimantation, sur la valeur des températures de Curie, sur la transition de phase ferromagnétique-paramagnétique. Puis nous mentionnerons quelques indications sur l'influence de l'ordre à courte distance sur les températures de Curie et l'aimantation moyenne.

\subsubsection{L'effet du désordre sur les propriétés magné-} tiques moyennes. - Un certain nombre de composés binaires ou ternaires ont pu être obtenus dans la phase cristalline et la phase amorphe. Il a été prouvé que le désordre structural tend à réduire l'aimantation à saturation. Mais cette réduction est très faible : $7 \%$ pour $\mathrm{Fe}_{75} \mathrm{P}_{6} \mathrm{~B}_{19}$ [18], quelques pour cent pour $\mathrm{CoSiB}$ et $\mathrm{FePC}$ [19]; dans le cas de $\mathrm{Co}_{3} \mathrm{~B}$ [20], le moment garde pratiquement la même valeur dans les deux phases. Pour les éléments purs comme le Ni, le $\mathrm{Co}$, le $\mathrm{Fe}$ et le $\mathrm{Gd}$, le moment dans l'état amorphe n'a pu être déterminé jusqu'ici que par extrapolation à partir d'alliages binaires ou ternaires. L'atténuation due au désordre pour le Co [21] et le Gd [22] semble être du même ordre que celle observée dans les composés. Le cas du Fer [23, 24] amorphe est encore l'objet de controverses, le moment du Fer dépendant de façon critique de la structure et de l'environnement.

Le moment des composés semi-métalliques (cristallins ou amorphes) faits de métaux de transition alliés avec $\mathrm{B}, \mathrm{C}, \mathrm{Si}, \mathrm{Ga}, \mathrm{Ge}$, As est réduit par rapport à celui des métaux de transition purs dans une proportion plus grande que ne le prévoit une loi de simple dilution. Cette réduction est communément attribuée à un transfert de charge des électrons s.p. du métalloïde vers les bandes $d$ partiellement vides du métal de 
transition. Cette description rend compte qualitativement des phénomènes tels que le déplacement d'ensemble des courbes de Slater Pauling pour l'amorphe Fe-P-B [25] comparées à celles du Fer pur cristallin, ou la variation du moment du Fer dans des composés ternaires amorphes comme Fe-P-C [26], Fe-P-B [27] lorsque l'on change les concentrations respectives des deux métalloïdes tout en gardant constante la concentration en Fer. Mais ce modèle de bandes rigides demeure phénoménologique (il a été discuté de façon critique par Alben et coll. [28]) et il ne permet pas de décrire quantitativement les phénomènes, en dépit des versions améliorées qui en ont été présentées récemment [29, 30]. Notons enfin que, pour expliquer l'augmentation de moment porté par le métal de transition dans des amorphes, des terres rares avec des métaux de transition, l'hypothèse a été émise d'une atténuation du transfert de charge sous l'effet du désordre [31]. En fait, le modèle du transfert de charge doit être confronté, pour chaque type d'alliage particulier, avec les résultats obtenus par photo-émission [32] ou par RMN sur les métalloïdes [33].

La dépendance en température de l'aimantation spontanée a été mesurée dans un certain nombre d'alliages ferromagnétiques amorphes. Les courbes obtenues (en coordonnées réduites) se sont avérées beaucoup plus plates que celles prédites par des fonctions de Brillouin. L'écart entre ces courbes pour Fe-P-C [34] et $\mathrm{Gd}_{4} \mathrm{Au}$ [22] et celles du Fer pur et du Gd pur dans l'état cristallin a été attribué à une distribution dans les intégrales d'échanges qui serait caractéristique des interactions à courte distance dans l'état amorphe. En suivant le formalisme de Handrich [35], l'aimantation spontanée en fonction de la température a été exprimée sous la forme suivante (en coordonnées réduites) :

$$
M(T)=1 / 2\left\{B_{\mathrm{S}}[(1+\bar{\delta}) x]+B_{\mathrm{S}}[(1-\bar{\delta}) x]\right\},
$$

où $x=[3 M(T) /(S+1)] T_{\mathrm{c}} / T$, et $\bar{\delta}$ exprime les déviations par rapport à une constante d'échange moyenne entre deux spins proches voisins dans un milieu désordonné : $\bar{\delta}^{2}=\left\langle\Delta J^{2}\right\rangle \mid\langle J\rangle^{2}$.

Les résultats expérimentaux interprétés selon ce modèle aboutissent à des valeurs de $\delta=0,3$ à 0,4 pour $\mathrm{FePC}$ et $\mathrm{Gd}_{4} \mathrm{Au}$. Ces valeurs et les présupposés du modèle de Handrich ont été critiqués récemment à partir d'études de la dépendance en température des différents pics d'absorption Mössbauer dans des alliages amorphes tels que $\mathrm{Fe}_{80} \mathrm{~B}_{20}$ [36] et FeBSi [37], et à partir de comparaisons avec des composés cristallins comme $\mathrm{Fe}_{3} \mathrm{Si}$. Les études concluent à la prédominance des interactions à longue distance dans ces alliages amorphes; la distribution des intégrales d'échanges serait donc faible, et l'effet du désordre se traduirait principalement par une distribution des effets de gradient de champ électrique. Des expériences récentes de $\mathrm{RMN}$ sur $\mathrm{Co}^{59}$ dilué dans FePB semblent conduire aux mêmes conclusions [38].
L'effet du désordre sur les valeurs des températures de Curie semble plus marqué que sur le moment à saturation à $0 \mathrm{~K}$. Ainsi, pour le système $\mathrm{Fe}_{75} \mathrm{P}_{6} \mathrm{~B}_{19}$, $T_{\mathrm{c}}$ dans l'état amorphe est réduit de $12 \%$ par rapport à la phase cristalline [18]. De même, on obtient par extrapolation une réduction de $15 \%$ pour $T_{\mathrm{c}}$ dans le $\mathrm{Gd}$ amorphe [22]. Cette réduction peut s'expliquer par le fait que la densité dans l'état amorphe est plus faible de quelques pour cent que celle mesurée dans le cristallin correspondant [39]. Par contre, les extrapolations donnent pour le Fer amorphe un $T_{\mathrm{c}}$ très faible : de 200 [40] à $300 \mathrm{~K}$ [18], ce qui semble indiquer que le Fer amorphe serait dans un état intermédiaire entre le Fer bcc et le Fer fcc.

Enfin, le problème de l'influence du désordre sur la transition de phase ferromagnétique-paramagnétique a été étudié dans plusieurs alliages amorphes à base de métaux de transition [41] et de Gd [22, 42]. Les exposants critiques déterminés par des mesures d'aimantation aux alentours de $T_{\mathrm{c}}$ vérifient les critères d'une transition de phase du second ordre, ce qui implique une valeur négative de l'exposant critique $\alpha$, selon les prédictions théoriques [43], et comme cela a été vérifié expérimentalement par des mesures de chaleur spécifique sur FePC [44]. Pour les amorphes à base de $\mathrm{Gd}$, les exposants critiques sont très voisins de ceux calculés dans un modèle de Heisenberg et observés pour le Gd polycristallin. On peut noter cependant une tendance pour le coefficient $\beta$ dans les amorphes étudiés à prendre des valeurs légèrement augmentées $(\beta \geqslant 0,40)$ par rapport à celles déterminées dans des systèmes cristallins voisins. Cette tendance peut s'expliquer dans un modèle de spins en défaut proposé par Müller-Krumbhaar [45].

2.2.2 Effets de l'ordre à courte distance sur les propriétés magnétiques moyennes. - De la dépendance en concentration de l'aimantation moyenne et de la température de Curie, il est possible de conclure à des changements d'ordre local qui ont échappé jusqu'ici aux études de structure par rayons $\mathrm{X}$ et qui sont confirmés par des études locales ( $R M N$, neutrons, Mössbauer). La première étude de ce type a été faite $[18,27]$ sur les alliages amorphes $\mathrm{Fe}_{79} \mathrm{P}_{21-x} \mathrm{~B}_{x}$ comparés aux composés cristallins $\mathrm{Fe}_{75} \mathrm{P}_{25-x} \mathrm{~B}_{x}$ [46]. Dans le système cristallin, on observe pour l'aimantation et $T_{\mathrm{c}}$ des dépendances en concentration de Bore radicalement différentes selon que le composé ternaire est de structure de type $\varepsilon\left(\mathrm{Fe}_{3} \mathrm{P}\right)$ ou de type $\varepsilon_{1}$ $\left(\mathrm{Fe}_{3} \mathrm{~B}\right)$. Les mêmes changements de pente pour $T_{\mathrm{c}}(x)$ et $\mu(x)$ interviennent dans le système amorphe, ce qui indique que l'ordre local est d'abord de type $\varepsilon$, puis devient de type $\varepsilon_{1}$ pour les plus fortes concentrations de Bore. Ceci a été confirmé par des mesures de spectroscopie Mössbauer qui ont mis en évidence deux types de distribution de champs hyperfins correspondant aux deux types d'ordre à courte distance [47]. De semblables changements d'ordre local ont été mis en évidence dans les alliages $\mathrm{Fe}_{100-x} \mathrm{~B}_{x}$ pour $x \approx 18$ [24] à partir de mesures d'aimantation 


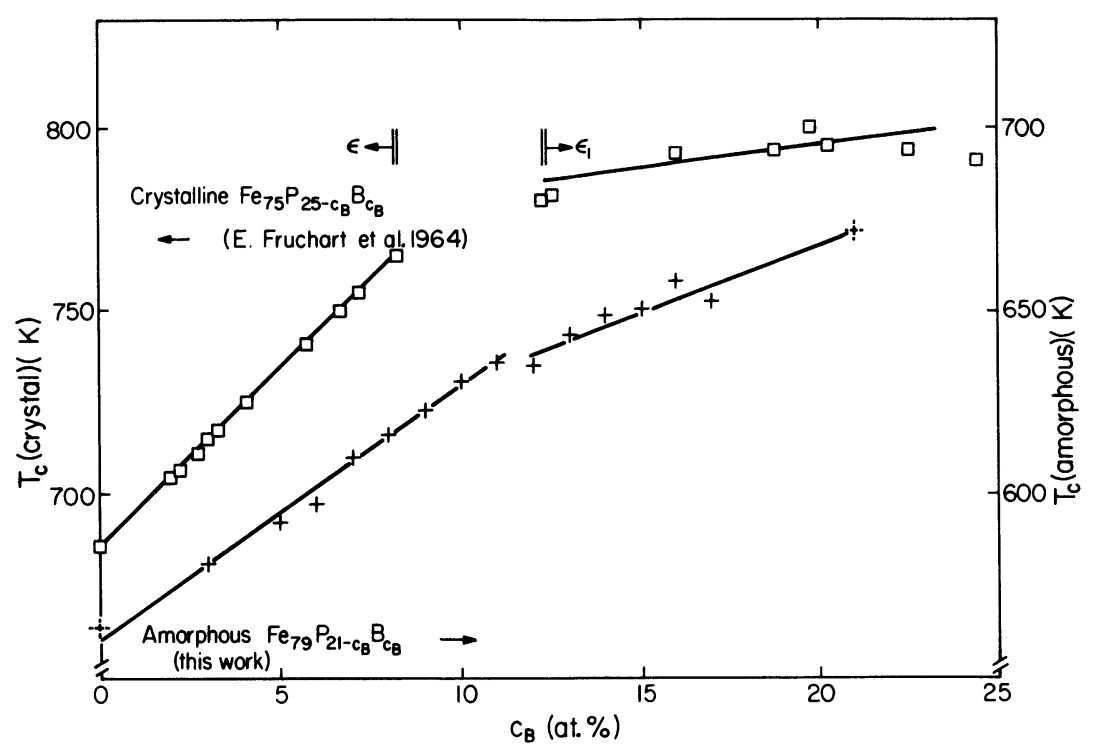

Fig. 3. - Variation de la température de Curie $T_{\mathrm{c}}$ en fonction de la concentration en Bore $\left(c_{\mathrm{B}}\right)$ dans les alliages amorphes $\mathrm{Fe}_{79} \mathrm{P}_{21-c_{\mathrm{B}}} \mathrm{B}_{c_{\mathrm{B}}}$ et dans les composés cristallins $\mathrm{Fe}_{75} \mathrm{P}_{25-c_{B}} \mathbf{B}_{c_{B}}$ (référence [18]).

[Curie temperature versus boron concentration in amorphous $\mathrm{Fe}_{79} \mathrm{P}_{21-c_{B}} \mathrm{~B}_{c_{B}}$ alloys and in crystalline $\mathrm{Fe}_{75} \mathrm{P}_{25-c_{B}} \mathrm{~B}_{c_{B}}$ compounds (reference [18]).]

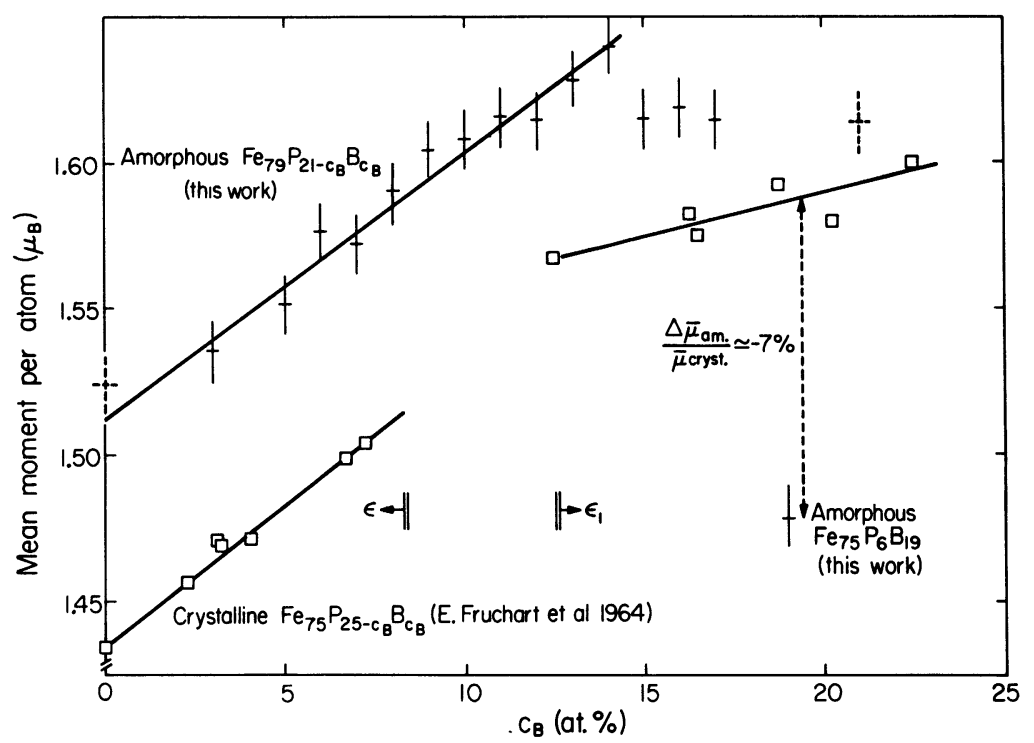

Fig. 4. - Variation du moment moyen par atome en fonction de la teneur en Bore $c_{\mathrm{B}}$ dans les alliages amorphes $\mathrm{Fe}_{79} \mathrm{P}_{21-c_{\mathrm{B}}} \mathrm{B}_{c_{\mathrm{B}}}$ et les composés cristallins $\mathrm{Fe}_{75} \mathrm{P}_{25-c_{B}} \mathrm{~B}_{c_{B}}$ (référence [18]).

[Mean moment per atom of alloy as a function of boron concentration in amorphous $F_{79} P_{21-c_{B}} B_{c_{B}}$ alloys and in crystalline $F_{7_{55}} P_{25-c_{B}} B_{c_{B}}$ compounds (reference [18]).]

et de $T_{\mathrm{c}}$, ainsi que dans les alliages $\mathrm{Fe}_{100-x} \mathrm{P}_{x}$ pour $x \approx 18$ at. $\%$ [48] et dans le système FeBSi [49] pour des concentrations de Fer de l'ordre de $75 \%$.

3. Les alliages amorphes contenant des terres rares dans un état ionique non S. - Dans les alliages amorphes à base de métaux de transition, les effets de gradients de champ électrique sont habituellement négligés (sans que cela d'ailleurs soit pleinement justifié). Dans le cas des ions non-S de terres rares, les effets de champ cristallin jouent à l'évidence un grand rôle. Ces effets ont été mis en évidence expéri- mentalement dans les amorphes du type $\mathrm{TbFe}_{2}$ [50], et ils ont été décrits dans l'approximation d'anisotropie aléatoire uniaxiale [51]. Dans ce modèle, les gradients de champ électrique dans un amorphe contribuent à l'Hamiltonien du système sous la forme : $-D J_{z}^{2}$, où $D$, la force d'anisotropie est supposée constante et positive, et où les axes de facile aimantation $z_{\mathrm{i}}$ varient de site en site de façon aléatoire. Récemment, Fert et Campbell [52] ont montré que cette approximation s'avère suffisante pour les terres rares lourdes ( $J$ grand), mais que dans le cas des valeurs de $J$ relativement petites, spécialement dans le cas 


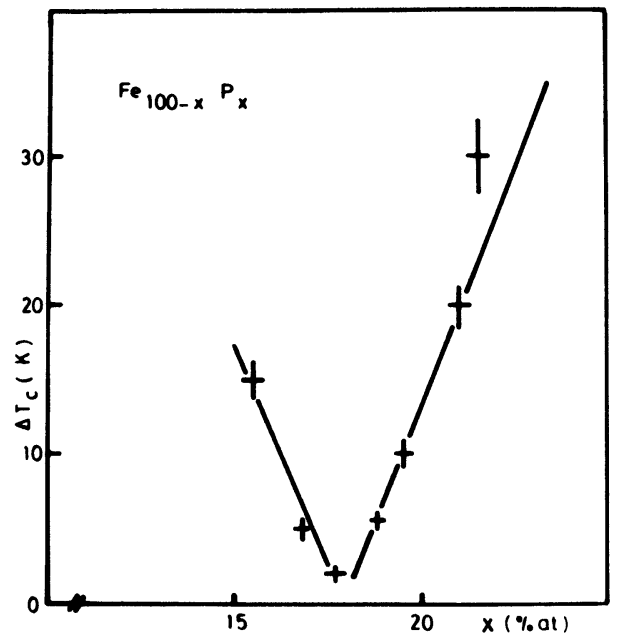

Fig. 5. - Largeur de la transition de Curie $\Delta T_{\mathrm{c}}$ en fonction de la concentration en Phosphore dans les alliages amorphes Fe-P obtenus par électro-déposition (J. Durand, non publié). [Les études de structure faites sur les mêmes échantillons à partir de diffraction aux rayons $\mathrm{X}$ ne mettent pas en évidence cet effet de concentration, cf. J. Logan, Phys. Status Solidi (A) 32 (1975) 361.]

[Curie transition width $\left(\Delta T_{\mathrm{c}}\right)$ versus phosphorus concentration in amorphous electrodeposited Fe-P alloys (J. DURAND, unpublished results). [Structural studies carried out from $\mathrm{X}$ ray diffraction on the same samples failed to exhibit any concentration effect, cf. J. Logan, Phys. Status Solidi (A) 32 (1975) 361.]]

d'ions non-Kramers, les termes non axiaux $\left(J_{x_{i}}^{2}, J_{y_{i}}^{2}\right)$ doivent être pris en compte. Nous présentons brièvement les informations que des mesures d'aimantation et de susceptibilité peuvent fournir sur les gradients de champ électrique dans un milieu amorphe.

Pour les systèmes contenant des terres rares faiblement couplées par échange ( $\mathrm{Ce}, \mathrm{Pr}, \mathrm{Nd})$, la dépendance en température de la susceptibilité reflète les effets de champ cristallin dans la limite des basses

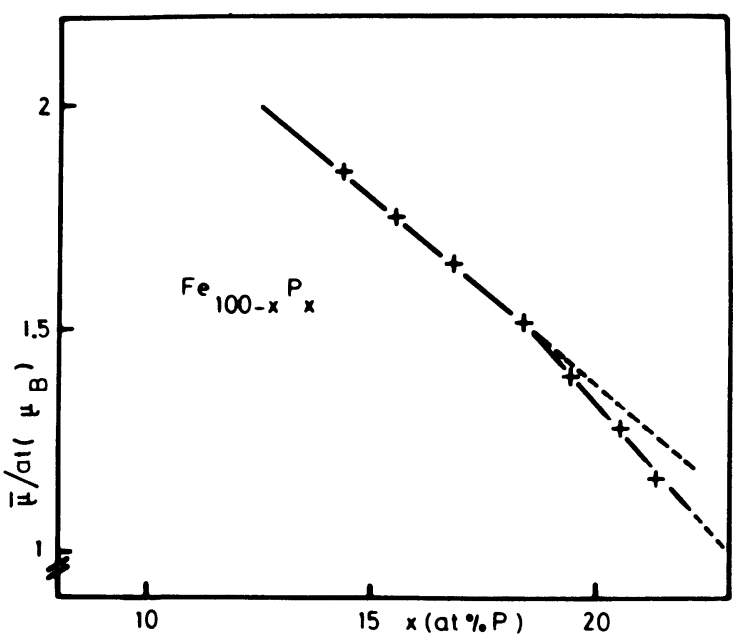

Fig. 6. - Moment moyen par atome de Fer en fonction de la concentration en Phosphore dans les alliages amorphes Fe-P (J. DuRAND, non publié).

[Mean magnetic moment per $\mathrm{Fe}$ atom versus phosphorus concentration in amorphous electrodeposited Fe-P alloys (J. DURAND, unpublished results).]

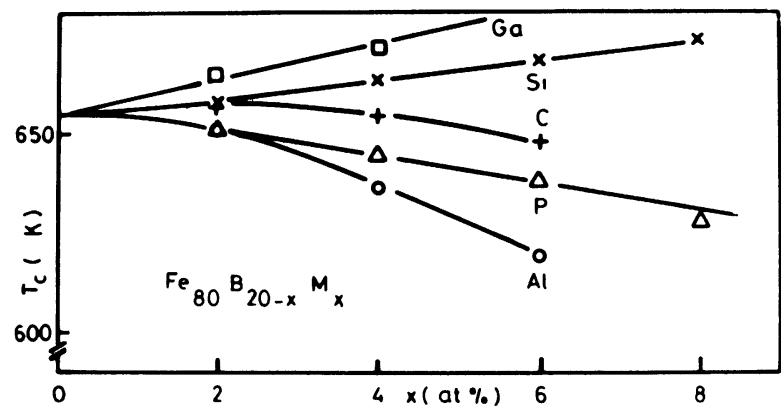

Fig. 7. - Température de Curie en fonction de la concentration en élément $\mathbf{M}(\mathbf{M}=\mathbf{P}, \mathrm{C}, \mathrm{Si}, \mathrm{Al}, \mathrm{Ga})$ dans les alliages amorphes $\mathrm{Fe}_{80} \mathrm{~B}_{20-x} \mathrm{M}_{x}$ (J. DURAND, non publié).

[Curie temperature versus $\mathrm{M}$ concentration $(\mathrm{M}=\mathrm{P}, \mathrm{C}, \mathrm{Si}, \mathrm{Al}, \mathrm{Ga}$ ) in amorphous $\mathrm{Fe}_{80} \mathrm{~B}_{20-x} \mathrm{M}_{x}$ alloys (J. DURAND, unpublished results).]

températures. A haute température $(T \geqslant 150 \mathrm{~K})$, la susceptibilité suit une loi de Curie-Weiss avec une constante correspondant à la valeur théorique d'un ion trivalent. Au-dessous de $150 \mathrm{~K}$, la susceptibilité s'écarte progressivement d'un comportement ionique. La constante de Curie-Weiss ainsi obtenue à basse température est atténuée d'un rapport $\alpha$ par rapport à la valeur de l'ion trivalent. D'après le modèle uniaxial, le rapport $\alpha$ est égal à $J /(J+1)$ pour $D>0$. Une symétrie planaire $(D<0)$ donnerait

$$
\alpha=1 / 2\left[(J+1)^{2}+1 / 4\right] / J(J+1)
$$

pour un ion Kramers, et une susceptibilité de Van Vleck indépendante de la température à basse température pour un ion non-Kramers [52]. D'autre part, la susceptibilité à haut champ fournit une estimation de la force d'anisotropie $D$ [53]. Il est donc possible de tester, de par la susceptibilité à

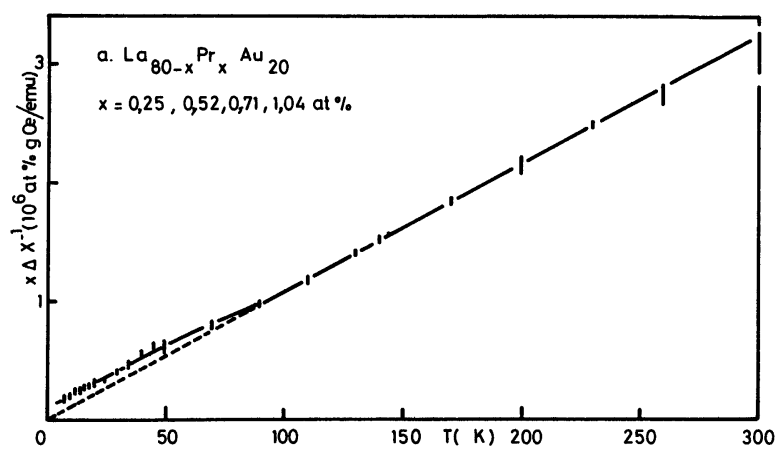

Fig. 8. - Inverse de l'augmentation de susceptibilité en fonction de la température pour du Pr dilué $(x \leqslant 1$ at. \%) dans les alliages amorphes $\mathrm{La}_{80-x} \mathrm{Pr}_{x} \mathrm{Au}_{20}$ (référence [55]). Pour $T \geqslant 100 \mathrm{~K}$, la susceptibilité prend des valeurs très proches

$$
\left(\mu_{\text {eff }}=(3,35 \pm 0,10) \mu_{\mathbf{B}} / \operatorname{Pr} \text { at. }\right)
$$

de la valeur ionique $\left(\mu_{\text {eff }}^{\mathrm{P}^{3+}}=3,57 \mu_{\mathrm{B}}\right)$.

[Inverse of incremental susceptibility as a function of temperature for $\operatorname{Pr}$ diluted $\left(x \leqslant 1\right.$ at. \%) in amorphous $\mathrm{La}_{80-x} \operatorname{Pr}_{x} \mathrm{Au}_{20}$ alloys (reference [55]). For $T \geqslant 100 \mathrm{~K}$, the susceptibility values are rather close $\left(\mu_{\text {eff }}=(3.35 \pm 0.10) \mu_{\mathbf{B}} / \operatorname{Pr}\right.$ at. $)$ to the theoretical ionic values $\left(\mu_{\text {eff }}^{\mathrm{Pr}^{3}+}=3.57 \mu_{\mathrm{B}}\right)$.] 


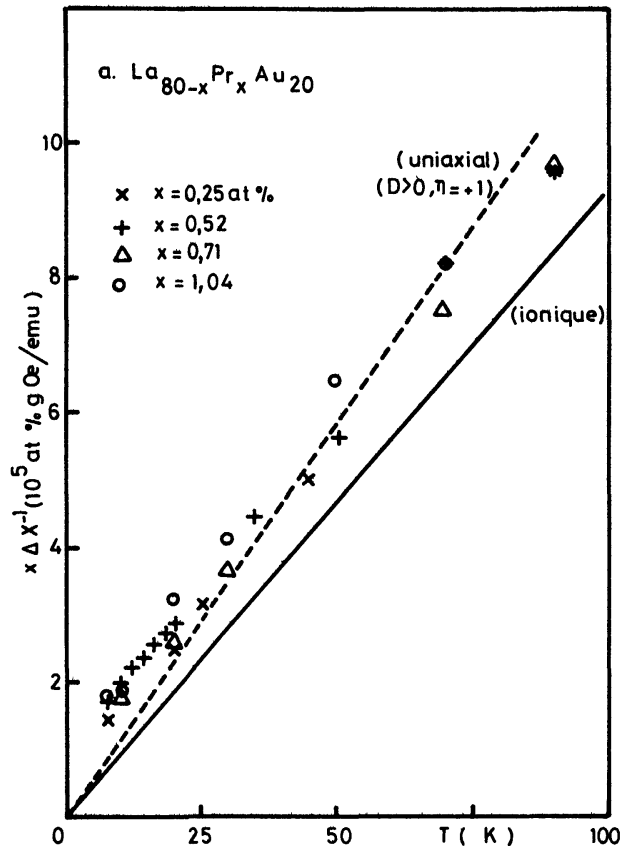

Fig. 9. - Extension de la figure 8 aux basses températures et comparaison avec les valeurs ioniques et celles prédites par le modèle d'anisotropie uniaxiale (référence [55])

[Extended scale of figure 8 for the low temperature range. Comparison is made with ionic values together with uniaxial anisotropy model predictions (reference [55]).]

basse température, la symétrie locale autour des ions de terres rares légères. Les résultats obtenus pour le $\mathrm{Ce}$ [54], le $\mathrm{Pr}$ et le $\mathrm{Nd}$ [55] dilués dans le $\mathrm{La}_{80} \mathrm{Au}_{20}$ montrent des écarts à la symétrie uniaxiale, qui doivent être attribués à des termes non axiaux.

Dans les systèmes amorphes contenant des terres rares lourdes, l'échange et l'anisotropie sont du même ordre de grandeur; d'où l'absence de saturation à haut champ, de larges champs coercitifs, de la rémanence, du traînage magnétique. Ces divers phénomènes, qui ont été analysés en détail pour le $\mathrm{Tb}-\mathrm{Ag}$ amorphe [56], ne sont pas propres à l'état amorphe. Ils ont été observés aussi dans des systèmes cristallins de basse symétrie où les spins ne sont pas colinéaires [57]. Il semble hors de doute cependant que le désordre structural contribue à les amplifier.

Notons enfin que les mesures magnétiques permettent d'identifier les états de valence dans les systèmes amorphes contenant des ions à valence anormale ou instable ( $\mathrm{Sm}, \mathrm{Eu})$.

4. Conclusion. - La mesure des propriétés magnétiques moyennes d'un alliage amorphe permet d'apprécier l'effet du désordre, et dans certains cas fournit des informations sur l'ordre local que des mesures de diffraction aux rayons $\mathrm{X}$ se sont avérées impuissantes à donner. Dans la plupart des cas, cependant, des mesures locales (Mössbauer, RMN, neutrons) sont nécessaires pour attester et préciser les renseignements obtenus par l'étude des propriétés magnétiques moyennes.

\section{References}

[1] Hasegawa, R. and Tsuei, C. C., Phys. Rev. B 3 (1971) 214.

[2] Sharon, T. E. and Tsuei, C. C., Phys. Rev. B 5 (1972) 1047.

[3] Hasegawa, R., J. Phys. Chem. Solids 32 (1971) 2487; J. Appl. Phys. 41 (1970) 4096.

[4] АмамоU, A., IEE Trans. Mag. 12 (1976) 948 ; id. in Amorphous Magnetism II, edit. Levy and Hasegawa (Plenum Press, New York, 1977) p. 265 ; Durand J., ibid. p. 305.

[5] Szofran, F. R., Gruzalski, G. R., Weymouth, J. W. Sellmyer, D. J. and Giessen, B. C., Phys. Rev. B 14 (1976) 1160

[6] Poon, S. J. and Durand, J., Phys. Rev. B 18 (1978) 6253.

[7] Durand, J. and Poon, S. J., J. Physique Colloq. 39 (1978) C 6.

[8] Poon, S. J. and Durand, J., Solid State Commun. 21 (1977) 999.

[9] Poon, S. J. and Durand, J., Solid State Commun. 21 (1977) 793.

[10] De Gennes, P. G., J. Physique Radium 23 (1962) 630;

Madhukar, A., J. Physique Colloq. 35 (1974) C4-295;

Kaneyoshi, T., J. Phys. F 5 (1975) 1014.

[11] Poon, S. J., Phys. Rev. B 21 (1980) 343.

[12] Berrada, A., Lapierre, M. F., Loegel, B., Panissod, P. and Robert, C., J. Phys. F 8 (1978) 845.

[13] Beille, J., Lienard, A. and Rebouillat, J. P., J. Physique Colloq. 40 (1979) C5-256.

[14] Chappert, J., Arrese-Boggiano, R. and Coey, J. M. D., J. Mag. Mag. Mat. 7 (1978) 175.

[15] Mangin, P., Piecuch, M., Marchal, G. and Janot, C., J. Phys. F 8 (1978) 2085.

[16] Amamou, A. and Durand, J., Comm. Phys. 1 (1976) 191.

[17] LOEGEL, B. and DuRAND, J. (à paraître).

[18] DuRAND, J., IEEE Trans. Mag. 12 (1976) 945.
[19] Kazama, H., Kameda, M. and Masumoto, T., aIP Conf. Proc. 34 (1976) 307.

[20] Hasegawa, R. and Ray, R., J. Appl. Phys. 50 (1979) 1586.

[21] Durand, J. and Lapierre, M. F., J. Phys. F 6 (1976) 1185.

[22] Poon, S. J. and Durand, J., Phys. Rev. B 16 (1977) 316.

[23] Felsch, W., Z. Physik 219 (1969) 280.

[24] Hasegawa, R. and Ray, R., J. Appl. Phys. 49 (1978) 4174.

[25] Mizoguchi, T., Yamauchi, K. and Miyajima, H., in Amorphous Magnetism, edit. Hooper and de Graf (Plenum Press, New York, 1973) p. 325.

[26] Chen, H. S., Phys. Status Solidi (A) 17 (1973) 561.

[27] DURAND, J. and YUNG, M., in Amorphous Magnetism II, edit. Levy and Hasegawa (Plenum Press, New York, 1977) p. 275.

[28] Alben, R., Budnick, J. I. and Cargill, G. S., in Metallic Glasses (AMS, Metals Park, 1978) p. 304.

[29] Yamauchi, K. and Mizoguchi, T., J. Phys. Soc. Japan 39 (1975) 541.

[30] O'Handley, R. C. and Boudreaux, D. S., Phys. Status Solidi (A) 45 (1978) 607.

[31] Chappert, J., J. Physique Colloq. 40 (1979) C2-107.

[32] Amamou, A. and Krill, G., Solid State Commun. 31 (1979) 971.

[33] Aliaga-Guerra, D., Panissod, P. and Durand, J., Solid State Commun. 28 (1978) 745; •

Aliaga-Guerra, D., Durand, J., Johnson, W. L. and Panissod, P., Solid State Commun. 31 (1979) 487.

[34] Tsuei, C. C. and Lilienthal, H., Phys. Rev. B 13 (1976) 4899. 
[35] Handrich, K., Phys. Status Solidi 32 (1969) K 55; Phys. Status Solidi (B) $\mathbf{5 3}$ (1972) K 17.

[36] Balogh, J. and VinCZe, I., Solid State Commun. 25 (1978) 695.

[37] Schurer, P. J. and Morrish, A. H., Solid State Commun. 30 (1979) 21.

[38] Durand, J., Aliaga-Guerra, D., Panissod, P. and HaseGAWA, R., Joint Intermag. MMM Conf. (New York, juillet 1979) J. Appl. Phys. 50 (1979) 7668.

[39] Cargill, G. S., Solid State Phys. 30 (1975) 227.

[40] Alperin, H. A. et al., AIP Conf. Proc. 29 (1976) 186 ; Heiman, N. et al., ibid. p. 130.

[41] Mizoguchi, T. and Yamauchi, K., J. Physique Colloq. 35 (1974) C4-287 ;

Mizoguchi, T., AIP Conf. Proc. 34 (1976) 286 ;

Yamada, K., Ishikawa, Y., Endoh, Y. and Masumoto, T., Solid State Commun. 16 (1975) 1335 ;

Figueroa, E., Lundgren, C., Beckman, O. and Bhagat, S. M., ibid 20 (1976) 961

[42] Durand, J., RaJ, K., Poon, S. J. and Budnick, J. I., IEEE Trans. Mag. 14 (1978) 722.

[43] Brooks Harris, A., J. Phys. C 7 (1974) 1671;

Brooks Harris, A. and Lubensky, T. C., Phys. Rev. Lett. 33 (1974) 1540 ;

Lubensky, T. C. and Harris Brooks, A., AIP Conf. Proc. 24 (1975) 311
[44] Schowalter, L. J., Salamon, M. B., Tsuei, C. C. and Craven, R. A., Bull. Am. Phys. Soc. 22 (1977) 264.

[45] Muller-Krumbhaar, H., J. Phys. C 9 (1976) 345.

[46] Fruchart, E., Triquet, A. M. and Fruchart, M., Ann. Chim. (Paris) 9 (1964) 323.

[47] Raj, K., Amamou, A., Durand, J., Budnick, J. I. and HaseGAWA, R., in Amorphous Magnetism II, edit. Levy and Hasagawa (Plenum Press, New York, 1977) p. 221. [48] DuRAND, J. (à paraître).

[49] Narita, K., Yamasaki, J. and Fukunaga, H., IEEE Trans. Mag. 13 (1977) 1544.

[50] Rhyne, J. J., Schelleng, J. H., and Koon, N. C., Phys. Rev. B 10 (1974) 4672.

[51] Cochrane, R. W., Harris, R. and Zuckermann, M. J., Phys. Rep. 48 (1978) 1.

[52] Fert, A. and Campbell, I. A., J. Phys. F 8 (1978) 257.

[53] Asomoza, R., Campbell, I. A., Fert, A., Lienard, A. and Rebouillat, J. P., J. Phys. F 9 (1979) 349.

[54] Durand, J. and PoOn, S. J. (à paraître).

[55] Hassanain, N., Berrada, A., Durand, J. and Loegel, B., ICM, Münich (sept. 79) J. Mag. Mag. Mat. 17-19 (1980) 1377.

[56] Boucher, B., Phys. Status Solidi (A) 40 (1977) 187;

Boucher, B. and Barbara, B., J. Phys. F 9 (1979) 151

[57] Barbara, B. and Uehara, M., IEEE Trans. Mag. 12 (1976) 6. 